\title{
WEIGHTED ITERATES AND VARIANTS OF THE HARDY-LITTLEWOOD MAXIMAL OPERATOR
}

BY

\author{
M. A. LECKBAND AND C. J. NEUGEBAUER
}

\begin{abstract}
In a recent paper, M. A. Leckband and C. J. Neugebauer obtained a rearrangement inequality for a generalized maximal operator with respect to two measures. For an application they studied norm bounds for the iterated HardyLittlewood maximal operator with respect to two measures. In this paper this theory is further developed and other applications of the rearrangement inequality are obtained.
\end{abstract}

1. Let $\mu, \nu$ be two measures on $\mathbf{R}^{n}$, and let there be associated with each cube $Q \subset \mathbf{R}^{n}$ a function $\phi_{Q}$ supported in $Q$. We consider the maximal operator

$$
M f(x)=\sup \int f \phi_{Q} d \nu
$$

where the sup is extended over all $Q$ with center $x$. If $g_{\lambda}^{*}$ is the nonincreasing rearrangement of $g$ with respect to the measure $\lambda$, i.e., $g_{\lambda}^{*}(t)=\inf \{y: \lambda\{|g|>y\} \leqslant t\}$, and if $\Phi(t)=\sup _{Q}\left\{\mu(Q) \phi_{Q, \nu}^{*}(\mu(Q) t)\right\}$, then we have proved, in [6], the following theorem.

THEOREM 1. $(M f)_{\mu}^{*}(\xi) \leqslant A \int_{0}^{\infty} \Phi(t) f_{\nu}^{*}(t \xi) d t$.

From this rearrangement inequality it is easy to get general norm inequalities. In particular, Minkowski's integral inequality gives

$$
\|M f\|_{p, \mu} \leqslant A\left(\int_{0}^{\infty} \frac{\Phi(t)}{t^{1 / p}} d t\right)\|f\|_{p, \nu},
$$

and thus, if $\Phi \in L\left(p^{\prime}, 1\right)$ (see [2]) we get a weighted norm inequality. At this point Muckenhoupt's $A_{p}$-condition enters; thus, if $(u, v) \in A_{p}$, i.e., $\int_{Q} u\left(\int_{Q} v^{1-p^{\prime}}\right)^{p-1} \leqslant$ $C|Q|^{p}$ [7], and if $d \mu=u d x, d \nu=v d x, \phi_{Q}(x)=(1 /|Q|)\left(\chi_{Q}(x) / v(x)\right)$, then the above $M f(x)=\sup (1 /|Q|) \int_{Q} f(t) d t$, the familiar Hardy-Littlewood maximal function. We have proved in [6] that if $(u, v) \in A_{p}, 1<p<\infty$, then $\Phi_{u, v} \in L\left(p^{\prime}, \infty\right)$, and $u=v$ is in $A_{p}, 1<p<\infty$, if and only if $\Phi_{u} \in L\left(p^{\prime}, 1\right)$, where

$$
\Phi_{u, v}(t)=\sup _{Q}\left\{\frac{u(Q)}{|Q|}\left(\frac{\chi_{Q}}{v}\right)_{\nu}^{*}(\mu(Q) t)\right\}
$$

and $\Phi_{u}=\Phi_{u, u}$.

Received by the editors February 26, 1982.

1980 Mathematics Subject Classification. Primary 42B25. 
The $j$ th iterated Hardy-Littlewood maximal function $M_{j} f$ turns out to be crucial in the extrapolation problem, i.e., when does $\|M f\|_{p . u} \leqslant A_{p}\|f\|_{p, v}$ imply the existence of $\varepsilon>0$ so that $\|M f\|_{p-\varepsilon, u} \leqslant B\|f\|_{p-\varepsilon, v}$ ? We have shown in [6] that extrapolation is possible if $\left\|M_{j}\right\|=\mathfrak{E}\left(A^{j}\right)$ as $j \rightarrow \infty$, where $\left\|M_{j}\right\|$ is the norm of $M_{j}$ as an operator from $L_{i}^{p}$ to $L_{u}^{p}$.

Using a dense set of functions, namely those which are nowhere constant, we obtain (Lemma 2)

$$
M_{j+1} f(x) \leqslant B_{n}^{j} \sup _{x \in Q} \frac{1}{|Q|} \int_{Q}|f(y)| \frac{\log ^{j}\left(|Q| / \rho_{Q}(y)\right)}{j !} d y,
$$

where

$$
\rho_{Q}(y)=\inf \left\{t: y \in\left\{x \in Q:|f(x)| \geqslant\left(f \chi_{Q}\right) *(t)\right\}\right\} .
$$

From this we obtain (Theorem 2) that if $(u, v) \in A_{p}$, then for each $q>p$ there is a constant $0<A_{q}<\infty$ such that $\left\|M_{j} f\right\|_{q, u} \leqslant A_{q}^{j}\|f\|_{q, v}$. This then implies that extrapolation of $\|M f\|_{p, u} \leqslant B\|f\|_{p, v}$ is possible if and only if $\left\|M_{j}\right\|=\mathcal{O}\left(A^{j}\right)$ as $j \rightarrow \infty$ (Theorem 3).

At a fixed $p$, iteration may not be possible, i.e., if $\left\|M_{j} f\right\|_{p, u} \leqslant A\|f\|_{p, v}$ for some $j>0$, then $M_{j+1}$ may not be bounded on $L_{v}^{p}$. We will also study conditions under which iterations in this case are possible by estimating the associated $\Phi(t)$ (Theorem 4).

The inequality in Theorem 1 readily lends itself to studying restricted weak type. In particular, $\Phi \in L\left(p^{\prime}, \infty\right)$ shows that $M f$ is restricted weak type $(p, p)$, i.e., $\|M f\|_{p, \infty, \mu} \leqslant A\|f\|_{p, 1, \nu}$. This observation is used to give a simple proof of the weak type behavior of a generalization of a maximal operator recently studied by Stein [9] (Theorem 6). For the usual Hardy-Littlewood maximal operator we will see (Theorem 7) that $\|M f\|_{p, x, u} \leqslant C\|f\|_{p, 1, v}, 1<p<\infty$, if and only if $\Phi \in L\left(p^{\prime}, \infty\right)$. We believe that this characterization is easier to use than the one found by Kerman [5]. The paper concludes with some variants of Theorem 1 .

2. We will establish an inequality similar to Theorem 1 for the $j$ th iterated Hardy-Littlewood maximal operator $M_{j} f$. It will be convenient to define a "telescoping" maximal operator $\bar{M}_{j} f$ as follows. First, define

$$
\bar{M}_{1 Q} f(x)=\sup _{x \in Q_{1} \subset Q} \frac{1}{\left|Q_{1}\right|} \int_{Q_{1}}|f|, \quad \bar{M}_{j Q} f(x)=\sup _{x \in Q_{j} \subset Q} \frac{1}{\left|Q_{j}\right|} \int_{Q_{j}} \bar{M}_{j-1, Q^{\prime}} f,
$$

i.e.,

$$
\begin{aligned}
\bar{M}_{j Q} f(x)= & \sup _{x \in Q_{1} \subset Q} \frac{1}{\left|Q_{1}\right|} \int_{Q_{1}} \sup _{x_{2} \in Q_{2} \subset Q_{1}} \frac{1}{\left|Q_{2}\right|} \int_{Q_{2}} \\
& \quad \cdots \frac{1}{\left|Q_{j-1}\right|} \int_{Q_{j-1}} \sup _{x_{j} \in Q_{j} \subset Q_{j-1}} \frac{1}{\left|Q_{j}\right|} \int_{Q_{j}}|f(t)| d t d x_{j} \cdots d x_{2} .
\end{aligned}
$$

Let $\bar{M}_{1} f=M f$, and for $j \geqslant 2$, define

$$
\bar{M}_{j} f(x)=\sup _{x \in Q} \frac{1}{|Q|} \int_{Q} \bar{M}_{j-1, Q} f(t) d t .
$$


Lemma 1. Let $f \in L^{p}\left(\mathbf{R}^{n}\right)$ for some $p>1$. Then there is $C_{n}>0$ such that for a.e. $x$,

$$
M_{j} f(x) \leqslant C_{n}^{j} \bar{M}_{j} f(x), \quad j=1,2, \ldots
$$

Proof. The condition $p>1$ assures that for a.e. $x, M_{j} f(x)<\infty, j=1,2, \ldots$, and we will show that the lemma holds for all such $x$ 's. Assume the inequality is true for $j-1$ and $M_{j} f(x)<\infty$. Then

$$
\begin{aligned}
M_{j} f(x) & =M\left(M_{j-1} f\right)(x) \leqslant C_{n}^{j-1} M\left(\bar{M}_{j-1} f\right)(x) \\
& <C_{n}^{j-1}\left(\frac{1}{|Q|} \int_{Q} \bar{M}_{j-1} f(u) d u+\varepsilon\right)
\end{aligned}
$$

for some $Q$ containing $x$. We now let

$$
S=\left\{u \in Q: \frac{1}{2} M\left(\bar{M}_{j-1} f\right)(x) \leqslant \bar{M}_{j-1} f(u)<\infty\right\},
$$

and we note that

$$
M\left(\bar{M}_{j-1} f\right)(x) \leqslant \frac{2}{|Q|} \int_{S} \bar{M}_{j-1} f(u) d u+2 \varepsilon .
$$

For each $u \in S$ choose a cube $Q_{u}$ with $u \in Q_{u}$ and

$$
\bar{M}_{j-1} f(u) \leqslant \frac{1}{\left|Q_{u}\right|} \int_{Q_{u}} \bar{M}_{j-2, Q_{u}} f(t) d t+\varepsilon .
$$

Since $\sup _{u \in S}\left\{\left|Q_{u}\right|\right\}<\infty$, select $u_{0} \in S$ for which $l_{u_{0}} \geqslant \frac{1}{2} \sup _{u \in S} l_{u}$, where $l_{u}$ is the sidelength of $Q_{u}$.

There are now two cases: $3 Q \supset Q_{u_{0}}$ and $3 Q_{u_{0}} \supset Q$. Then $5 Q \supset Q_{u}$ and $5 Q_{u_{0}} \supset Q_{u}$ for $u \in S$, respectively. Thus, in the first case,

$$
M\left(\bar{M}_{j-1} f\right)(x) \leqslant \frac{2 \cdot 5^{n}}{|5 Q|} \int_{S} \sup _{t \in Q_{1} \subset 5 Q} \frac{1}{\left|Q_{1}\right|} \int_{Q_{1}} \bar{M}_{j-2, Q_{1}} f(y) d y d t+2 \varepsilon
$$

and, hence,

$$
M_{j} f(x) \leqslant \frac{2 \cdot 5^{n} C_{n}^{j-1}}{|5 Q|} \int_{5 Q} \bar{M}_{j-1,5 Q} f+2 \varepsilon \leqslant C_{n}^{j} \bar{M}_{j} f(x)+2 \varepsilon .
$$

In the second case we get for $z \in 5 Q_{u_{0}}$,

$$
\varepsilon+\sup _{z \in Q_{1} \subset 5 Q_{u_{0}}} \frac{5^{n}}{\left|Q_{1}\right|} \int_{Q_{1}} \bar{M}_{j-2, Q_{1}} f \geqslant \varepsilon+\frac{5^{n}}{\left|5 Q_{u_{0}}\right|} \int_{Q_{u_{0}}} \bar{M}_{j-2, Q_{u_{0}}} f \geqslant \frac{1}{2} M\left(\bar{M}_{j-1} f\right)(x),
$$

and thus,

$$
\begin{aligned}
M\left(\bar{M}_{j-1} f\right)(x) & \leqslant 2 \varepsilon+\frac{2 \cdot 5^{n}}{\left|5 Q_{u_{0}}\right|} \int_{5 Q_{u_{0}} z \in Q_{1} \subset 5 Q_{u_{0}}} \sup _{\left|Q_{1}\right|} \int_{Q_{1}} \bar{M}_{j-2, Q_{1}} f \\
& =2 \varepsilon+\frac{C_{n}}{\left|5 Q_{u_{0}}\right|} \int_{5 Q_{u_{0}}} \bar{M}_{j-1,5 Q_{u_{0}}} f \leqslant 2 \varepsilon+C_{n} \bar{M}_{j} f(x) .
\end{aligned}
$$

This completes the proof.

For $f: \mathbf{R}^{n} \rightarrow[0, \infty]$, let $f^{*}$ be the rearrangement of $f$ relative to Lebesgue measure on $\mathbf{R}^{n}$. We will also assume that $f$ is nowhere constant, i.e., $|\{x: f(x)=a\}|=0$, 
$a>0$. For a cube $Q \subset \mathbf{R}^{n}$, let $E_{t}=\left\{x \in Q: f(x) \geqslant\left(f \cdot \chi_{Q}\right)^{*}(t)\right\}$. Then $\left|E_{t}\right|=t$ and $t^{-1} \int_{0}^{t}\left(f \chi_{Q}\right)^{*}=t^{-1} \int_{E_{t}} f \chi_{Q}$.

LEMMA 2. Let $f: \mathbf{R}^{n} \rightarrow[0, \infty]$ be in $L^{p}\left(\mathbf{R}^{n}\right)$ for some $p>1$ and nowhere constant. Then there exists $B_{n}>0$ such that for a.e. $x$,

$$
M_{j+1} f(x) \leqslant B_{n}^{j} \sup _{x \in Q} \frac{1}{|Q|} \int_{Q} f(y) \frac{\log ^{j}\left(|Q| / \rho_{Q}(y)\right)}{j !} d y,
$$

where $\rho_{Q}(y)=\inf \left\{t: y \in E_{t}\right\}$.

Proof. By Lemma 1 , for a.e. $x, M_{j+1} f(x) \leqslant C_{n}^{j+1} \bar{M}_{j+1} f(x)$. By the $n$-dimensional version of Lemma 1 of [6] we see that

$$
\bar{M}_{j+1} f(x) \leqslant A_{n}^{j} \sup _{x \in Q} \frac{1}{|Q|} \int_{0}^{|Q|} \frac{1}{t_{1}} \int_{0}^{t_{1}} \cdots \frac{1}{t_{j}} \int_{0}^{t_{1}}\left(f \cdot \chi_{Q}\right)^{*}(\tau) d \tau d t_{j} \cdots d t_{1} .
$$

For the proof of the lemma we assume, for simplicity $j=2$. Then

$\frac{1}{|Q|} \int_{0}^{|Q|} \frac{1}{t} \int_{0}^{t} \frac{1}{s} \int_{0}^{s}\left(f \cdot \chi_{Q}\right)^{*}(u) d u d s d t$

$$
\begin{aligned}
& =\frac{1}{|Q|} \int_{0}^{|Q|} \frac{1}{t} \int_{0}^{t} \frac{1}{\left|E_{s}\right|} \int_{E_{t}} f(x) d x d s d t=\frac{1}{|Q|} \int_{0}^{|Q|} \frac{1}{t} \int_{E_{t}} f(x) \int_{\rho_{Q^{(}}(x)}^{t} \frac{1}{s} d s d x d t \\
& =\frac{1}{|Q|} \int_{0}^{|Q|} \frac{1}{t} \int_{E_{t}} f(x) \log \left(\frac{t}{\rho_{Q}(x)}\right) d x d t=\frac{1}{|Q|} \int_{Q} f(x) \frac{1}{2} \log ^{2}\left(\frac{|Q|}{\rho_{Q}(x)}\right) d x .
\end{aligned}
$$

Now let $(u, v)$ be a pair of weights with $u \geqslant 0$ in $L_{\text {loc }}^{1}\left(\mathbf{R}^{n}\right)$ and $0<v<\infty$, a.e. Set $d \mu=u d x, d \nu=v d x$.

LEMMA 3. With the same hypothesis as in Lemma 2,

$$
\left(M_{j+1} f\right)_{\mu}^{*}(\xi) \leqslant A B_{n}^{j} \int_{0}^{\infty} \Phi_{j}(t) f_{\nu}^{*}(t \xi) d t
$$

where

$$
\Phi_{j}(t)=\Phi_{j, f}(t)=\sup _{Q}\left\{\frac{\mu(Q)}{|Q|}\left(\frac{\log j\left(|Q| / \rho_{Q}(x)\right)}{j ! v(x)} \chi_{Q}(x)\right)_{\nu}^{*}(\mu(Q) t)\right\} .
$$

Proof. This is Theorem 1 with

$$
\phi_{Q}(x)=\frac{1}{|Q|} \frac{\log j\left(|Q| / \rho_{Q}(x)\right)}{j ! v(x)} \chi_{Q}(x)
$$

coupled with Lemma 2.

THEOREM 2. Let $(u, v)$ be a pair of weights as above and assume that $\|M f\|_{q, u} \leqslant$ $B_{q}\|f\|_{q, v}, 1 \leqslant p<q$. Then for each $q>p$ there is a constant $0<A_{q}<\infty$ such that $\left\|M_{j} f\right\|_{q, u} \leqslant A_{q}^{j}\|f\|_{q, v}$.

Proof. We may assume that $f$ is nowhere constant. We will estimate $\Phi_{j}(t)$ of Lemma 3 and show that $\Phi_{j} \in L\left(q^{\prime}, \infty\right), q>p$. Fix $q>p$, and let $p<p_{0}<q$. Since 
$\|M f\|_{p_{0} . u} \leqslant B_{p_{0}}\|f\|_{p_{0}, v}$, the pair $(u, v) \in A_{p_{0}}$, i.e..

$$
\mu(Q)\left(\int_{Q} v^{1-p_{i}^{\prime}}\right)^{p_{0}-1} \leqslant C|Q|^{p_{0}}
$$

We next note that

$$
\begin{aligned}
& \left(\frac{\log j\left(|Q| / \rho_{Q}(x)\right)}{j ! v(x)} \chi_{Q}(x)\right)_{v^{\prime}}^{*}(t) \leqslant \frac{1}{t^{1 / q^{\prime}}}\left(\int_{Q} \frac{\log { }^{1 q^{\prime}}(|Q| / \rho Q(x))}{j !^{\prime q^{\prime}}} v^{1-q^{\prime}} d x\right)^{1 / q^{\prime}} \\
& \leqslant \frac{1}{j ! t^{1 / q^{\prime}}}\left(\int_{Q} \log ^{1 q^{\prime} r^{\prime}}\left(\frac{|Q|}{\rho_{Q}(x)}\right) d x\right)^{1 / r^{\prime} q^{\prime}}\left(\int_{Q} v^{\left.1-p^{\prime}\right)}\right)^{1 / q\left(p_{(1)}^{\prime}-1\right)}
\end{aligned}
$$

where $r=\left(p_{0}^{\prime}-1\right) /\left(q^{\prime}-1\right)>1$. Since the rearrangement of $\log |Q| / \rho_{Q}(x)$ is $\log |Q| / t$, and $(1 / a) \int_{0}^{a} \log ^{r}(a / t) d t=\Gamma(r+1), r>-1$, the above equals

$$
\frac{\Gamma\left(j q^{\prime} r^{\prime}+1\right)^{1 / r^{\prime} q^{\prime}}}{t^{1 / q^{\prime}} j !}|Q|^{1 / r^{\prime} q^{\prime}}\left(\int_{Q} v^{1-p_{i}^{\prime}}\right)^{1 / q\left(p_{0}^{\prime}-1\right)} .
$$

Let $c_{j}=\Gamma\left(j q^{\prime} r^{\prime}+1\right)^{1 / r^{\prime} q^{\prime}} / j$ ! and observe that from Stirling's formula one gets $C_{j}^{1 / j} \leqslant C_{*}$, where $C_{*}$ is a constant depending on the product $q^{\prime} r^{\prime}$ only. All this gives

$$
\begin{gathered}
\frac{\mu(Q)}{|Q|}\left(\frac{\log ^{j}\left(|Q| / \rho_{Q}(x)\right)}{j ! v(x)} \chi_{Q}(x)\right)_{\nu}^{*}(\mu(Q) t) \\
\leqslant \frac{C_{*}^{j}}{t^{1 / q^{\prime}}} \cdot \frac{\mu(Q)^{1 / q}}{|Q|^{1-1 / r^{\prime} q^{\prime}}}\left(\int_{Q} v^{1-p_{(0}^{\prime}}\right)^{1 / q\left(p_{0}^{\prime}-1\right)} .
\end{gathered}
$$

We finally observe that $1-1 / r^{\prime} q^{\prime}=p_{0} / q,\left(p_{0}-1\right)\left(p_{0}^{\prime}-1\right)=1$, and thus $\Phi_{j}(t) \leqslant$ $C C_{*}^{j} / t^{1 / q^{\prime}}$.

We now complete the proof and fix $s>p$. Then from Lemma 3 we get

$$
\left\|M_{j+1} f\right\|_{s, u} \leqslant A B_{n}^{j}\left(\int_{0}^{\infty} \frac{\Phi_{j}(t)}{t^{1 / s}} d t\right)\|f\|_{s, v} .
$$

Now choose $p<q_{1}<s$ and $s<q_{2}<\infty$ and observe that

$$
\int_{0}^{\infty} \frac{\Phi_{j}(t)}{t^{1 / s}} d t=\int_{0}^{1}+\int_{1}^{\infty} \leqslant \int_{0}^{1} \frac{\alpha^{j}}{t^{1 / q_{1}^{\prime}+1 / s}} d t+\int_{1}^{\infty} \frac{\beta^{j}}{t^{1 / q_{2}^{\prime}+1 / s}} d t \leqslant \gamma^{j},
$$

and the proof of Theorem 2 is complete.

3. In [6] we have shown that if $\left\|M_{j} f\right\|_{p, u} \leqslant A_{j}\|f\|_{p, v}$ for some $1<p, j=1,2, \ldots$, and if

$$
\Phi(t)=\sup _{Q}\left\{\frac{\mu(Q)}{|Q|}\left(\frac{\chi_{Q}}{v}\right)_{\nu}^{*}(\mu(Q) t)\right\}
$$

then

$$
\Phi\left(2^{-N}\right) \leqslant C \frac{A_{j+1}}{B^{j}}\left(\frac{j !}{N^{j}}\right) 2^{N / p^{\prime}}
$$


From this and $A_{j}=\mathfrak{C}\left(A^{j}\right)$, one gets that $\Phi \in L\left((p-\varepsilon)^{\prime}, 1\right)$ for some $\varepsilon>0$, and thus $\|M f\|_{p-\varepsilon, u} \leqslant A\|f\|_{p-\varepsilon, v}$. All this was done in the context $n=1$, which we shall also assume for the next theorem.

Theorem 3. Let $(u, v) \in A_{p}$ for some $p>1$. Then there is $\varepsilon>0$ with $(u, v) \in A_{p-\varepsilon}$ if and only if $\sup _{\|f\|_{p, t}=1}\left\|M_{j} f\right\|_{p, u}=\hat{c}\left(A^{j}\right)$.

Proof. If $(u, v) \in A_{p-\varepsilon}$, the result follows from Theorem 2 , and the converse was just mentioned and is Theorem 6 in [6].

There are examples which show that the norm inequality $\|M f\|_{p, u} \leqslant A\|f\|_{p, v}$ does not admit an iteration (e.g. Theorem 4 in [6]).

If we let

$$
\Phi_{0}(t)=\sup \left\{\frac{\mu(Q)}{|Q|}\left(\frac{\chi_{Q}}{v}\right)_{\nu}^{*}(\mu(Q) t)\right\},
$$

the next theorem gives an estimate of $\Phi_{j}(t)$ in terms of $\Phi_{0}(t)$ that may allow an iteration up to a certain index.

THEOREM 4. Let $f: \mathbf{R}^{n} \rightarrow[0, \infty]$ be in $L^{p}\left(\mathbf{R}^{n}\right)$ for some $p>1$ and nowhere constant. Then

$$
\Phi_{j}\left(2^{-N}\right) \leqslant C\left[\frac{N^{j}}{j !} \Phi_{0}\left(\frac{2^{-N}}{2}\right)+N^{j}\right], \quad N=1,2, \ldots
$$

Proof. For a fixed $N$ consider

$$
L_{Q, j}=\frac{\mu(Q)}{|Q|}\left(\frac{\log ^{j}\left(|Q| / \rho_{Q}(x)\right)}{j ! v(x)} \chi_{Q}(x)\right)_{\nu}^{*}\left(\mu(Q) 2^{-N}\right) .
$$

Let $Q_{N}=\left\{x \in Q: \log \left(|Q| / \rho_{Q}(x)\right)>N / \log _{2} e\right\} \quad$ or $\quad Q_{N}=\left\{x \in Q: \rho_{Q}(x)<\right.$ $\left.\mid Q 2^{-N}\right\}$. We note that $\left|Q_{N}\right|=|Q| 2^{-N}$ and thus

$$
\begin{aligned}
L_{Q, j} \leqslant & C \frac{\mu(Q)}{|Q|}\left(\frac{N^{j}}{j ! v(x)} \cdot \chi_{Q \backslash Q_{N}}(x)\right)_{\nu}^{*}\left(\mu(Q) \frac{2^{-N}}{2}\right) \\
& +\frac{\mu(Q)}{|Q|}\left(\frac{\log ^{j}\left(|Q| / \rho_{Q}(x)\right)}{j ! v(x)} \chi_{Q_{N}}(x)\right)_{\nu}^{*}\left(\mu(Q) \frac{2^{-N}}{2}\right) .
\end{aligned}
$$

The first expression on the right is at most $\left(N^{j} / j !\right) \Phi_{0}\left(2^{-N-1}\right)$. The second term is zero if $\nu\left(Q_{N}\right) \leqslant \mu(Q) 2^{-N-1}$. Hence we assume that $\nu\left(Q_{N}\right)>\mu(Q) 2^{-N-1}$ and construct a set $S_{N} \subset Q_{N}$ such that if we let

$$
\alpha_{N}=\left(\frac{\log ^{j}\left(|Q| / \rho_{Q}(x)\right)}{j ! v(x)} \chi_{Q_{N}}\right)_{\nu}^{*}\left(\mu(Q) 2^{-N-1}\right),
$$

then

(i)

$$
\frac{1}{2} \mu(Q) 2^{-N-1} \leqslant \nu\left(S_{N}\right) \leqslant \mu(Q) 2^{-N-1},
$$


(ii)

$$
\frac{\log ^{j}\left(|Q| / \rho_{Q}(x)\right)}{j ! v(x)} \geqslant \alpha_{N} \quad \text { for } x \in S_{N}
$$

From this we get

$$
\begin{aligned}
\alpha_{N} \nu\left(S_{N}\right) & \leqslant \int_{Q_{N}} \frac{\log ^{j}\left(|Q| / \rho_{Q}(x)\right)}{j !} d x \leqslant \int_{0}^{\left|Q_{N}\right|} \frac{\log ^{j}(|Q| / t)}{j !} d t \\
& =\left|Q_{N}\right| \sum_{k=0}^{j} \frac{\log ^{k}\left(|Q| /\left|Q_{N}\right|\right)}{k !} \leqslant C\left|Q_{N}\right| \log ^{j}\left(\frac{|Q|}{\left|Q_{N}\right|}\right) .
\end{aligned}
$$

Thus

$$
\alpha_{N} \leqslant C \frac{\left|Q_{N}\right| \log ^{j}\left(|Q| /\left|Q_{N}\right|\right)}{\nu\left(S_{N}\right)} \text { and } \frac{\mu(Q)}{|Q|} \alpha_{N} \leqslant C N^{j},
$$

and the proof is complete.

Corollary. Let $(u, v) \in A_{p}$ for some $1<p<\infty$. If $\Phi_{0}\left(2^{-N}\right) \leqslant\left(C / N^{k}\right) 2^{N / p^{\prime}}$, $N=1,2, \ldots$, then $\left\|M_{j} f\right\|_{p, u} \leqslant A_{j}\|f\|_{p, v}, j=1,2, \ldots, k-1$, and

$$
\mu\left\{x: M_{k} f(x)>y\right\} \leqslant\left(C / y^{p}\right)\|f\|_{p, v}^{p} .
$$

Proof. From Lemma 3 and Minkowski’s integral inequality we get

$$
\left\|M_{k-1}\right\|_{p, u} \leqslant C\left(\int_{0}^{\infty} \frac{\Phi_{k-2}(t)}{t^{1 / p}} d t\right)\|f\|_{p, v} .
$$

We write $\int_{0}^{\infty}=\int_{0}^{1}+\int_{1}^{\infty}$, and to estimate $\int_{0}^{1}$ we use Theorem 4 and note that

$$
\Phi_{k-2}\left(2^{-N}\right) \leqslant C\left(\frac{2^{N / p^{\prime}}}{N^{2}}+N^{k-2}\right) .
$$

From this, $\sum \Phi_{k-2}\left(2^{-N}\right) / 2^{N / p^{\prime}}<\infty$ and $\Phi_{k-2} \in L\left(p^{\prime}, 1\right)$ on $[0,1]$.

For the integral $\int_{1}^{\infty}$ we use the proof in Theorem 2, where it was shown that $\Phi_{k-2} \in L\left(q^{\prime}, \infty\right), q>p$. Thus

$$
\int_{1}^{\infty} \frac{\Phi_{k-2}(t)}{t^{1 / p}} d t \leqslant C \int_{1}^{\infty} \frac{d t}{t^{1 / q^{\prime}+1 / p}}<\infty .
$$

The weak type estimate follows again from Lemma 3 by noting that by Hölder's inequality,

$$
\left(M_{k} f\right)_{\mu}^{*}(\xi) \leqslant C\left\|\Phi_{k-1}\right\|_{p^{\prime}} \cdot\|f\|_{p, \nu} \cdot 1 / \xi^{1 / p}
$$

Finally,

$$
\left\|\Phi_{k-1}\right\|_{p^{\prime}}^{p^{\prime}}=\int_{0}^{\infty} \Phi_{k-1}^{p^{\prime}} d t=\int_{0}^{1}+\int_{1}^{\infty}
$$

Now

$$
\int_{0}^{1} \Phi_{k-1}^{p^{\prime}} d t \leqslant C \sum \Phi_{k-1}^{p^{\prime}}\left(2^{-N}\right) 2^{-N}
$$


and, from Theorem $4, \Phi_{k-1}^{p^{\prime}}\left(2^{-N}\right) \leqslant C\left(2^{N} / N^{p^{\prime}}+N^{p^{\prime}(k-1)}\right)$. Hence $\int_{0}^{1}<\infty$. As before, $\int_{1}^{\infty} \leqslant C \int_{1}^{\infty} d t / t^{p^{\prime}} / q^{\prime}<\infty$, since $q>p$.

REMARK. The above corollary can be viewed as the converse of Theorem 5 in [6].

4. In this section we will show how Theorem 5 of [6] can be used to obtain extrapolation results from the magnitude of $\left\|M_{j}\right\|$. We assume $n=1$, the setting in which Theorem 5 of [6] has been proved.

From Theorem 2 we have that if $\|M f\|_{q, u} \leqslant B_{q}\|f\|_{q, v}, q>p>1$, then $\sup _{\|f\|_{q, v}=1}\left\|M_{j} f\right\|_{q, u}=\theta\left(A_{q}^{j}\right)$.

THEOREM 5. Let $1<p<\infty$. There is a constant $C_{p}>0$ such that the following holds. If for some $r_{0}>p, A_{r_{0}} \leqslant C_{p} /\left(r_{0}-p\right)$, then $\left\|M_{j} f\right\|_{p, u} \leqslant B_{j}\|f\|_{p, v}, j=1,2, \ldots$

Proof. From Theorem 5 in [6] we have constants $C>0, B>0$ so that for $r>p$,

$$
\begin{aligned}
\Phi_{0}\left(2^{-N}\right) & \leqslant C A_{r}\left(B A_{r}\right)^{j}\left(\frac{j !}{N^{j}}\right) 2^{N(1 / p-1 / r)} \cdot 2^{N / p^{\prime}} \\
& \leqslant C A_{r}\left(\frac{B A_{r} \cdot j}{e N}\right)^{j} j^{1 / 2} 2^{N(1 / p-1 / r)} \cdot 2^{N / p^{\prime}}
\end{aligned}
$$

by Stirling's formula. Hence, if $\alpha_{r}=e / 2 B A_{r}, j=\left[\alpha_{r} N\right]$, we get

$$
\Phi_{0}\left(2^{-N}\right) \leqslant C A_{r}\left(\frac{1}{2}\right)^{\alpha_{r} N}\left(\alpha_{r} N\right)^{1 / 2} 2^{N(1 / p-1 / r)} \cdot 2^{N / p^{\prime}} .
$$

Hence, from Theorem 4 ,

$$
\Phi_{k}\left(2^{-N}\right) \leqslant C\left(\frac{N^{k}}{k !} A_{r}\left(\frac{1}{2}\right)^{\alpha_{r} N}\left(\alpha_{r} N\right)^{1 / 2} 2^{N(1 / p-1 / r)} \cdot 2^{N / p^{\prime}}+N^{k}\right) .
$$

Now let $C_{p}=e p^{2} / 2 B$, and let $r_{0}>p$, for which $\left(r_{0}-p\right) A_{r_{0}} \leqslant C_{p}$. Then

$$
\alpha_{r_{0}} \geqslant \frac{r_{0}-p}{p^{2}}>\frac{r_{0}-p}{r_{0} p}=\frac{1}{p}-\frac{1}{r_{0}} .
$$

We claim now that

$$
A_{r_{0}}\left(\alpha_{r_{0}} N\right)^{1 / 2} 2^{N\left(1 / p-1 / r_{0}\right)} \leqslant N^{-k-2} 2^{\alpha_{r_{0}} N}, \quad N \geqslant N_{0} .
$$

With $\log$ to the base 2 this is

$$
\frac{1}{N} \log A_{r_{0}}+\frac{1}{N} \log \left(\sqrt{\alpha_{r_{0}} N} N^{2+k}\right)+\left(\frac{1}{p}-\frac{1}{r_{0}}\right) \leqslant \alpha_{r_{0}} .
$$

Since $\alpha_{r_{0}}>1 / p-1 / r_{0}$, this is possible for $N \geqslant N_{0}$. Hence, $\sum \Phi_{k}\left(2^{-N}\right) / 2^{N / p^{\prime}}<\infty$ and $\Phi_{k} \in L\left(p^{\prime}, 1\right)$ on $[0,1]$. From Theorem $2, \Phi_{k} \in L\left(q^{\prime}, \infty\right), q>p$, from which $\Phi_{k} \in L\left(p^{\prime}, 1\right)$ on $[1, \infty)$. Minkowski's integral inequality applied to Lemma 3 now completes the proof.

5. We will show in this section how Theorem 1 can be used to study the restricted weak type behavior of a general maximal operator. From Theorem 1 one obtains from $\Phi \in L\left(p^{\prime}, \infty\right)$ that

$$
(M f)_{\mu}^{*}(\xi) \leqslant B \int_{0}^{\infty} \frac{f_{\nu}^{*}(t \xi)}{t^{1 / p^{\prime}}} d t=\frac{B}{\xi^{1 / p}}\|f\|_{p, 1, \nu} .
$$


This is the same as $\|M f\|_{p, \infty, \mu} \leqslant B\|f\|_{p, 1, \nu}$, or $M f$ is restricted weak type $(p, p)$. With this observation it will be easy to obtain the weak type behavior of a maximal operator generalizing the one recently studied by E. M. Stein [9].

Theorem 6. Let $\mu>0, \nu \geqslant 0$ be two Borel measures. Let $1 \leqslant q \leqslant p$, and let $M_{p q} f(x)=\sup \left\|f \chi_{Q}\right\|_{p, q, v} /\left\|\chi_{Q}\right\|_{p, q, \mu}$, where the sup is extended over all cubes centered at $x$. Then

$$
\left\|M_{p q} f\right\|_{p, \infty, \mu} \leqslant A\|f\|_{p, q, \nu} \text { or } \quad \mu\left\{x: M_{p q} f(x)>y\right\} \leqslant\left(C / y^{p}\right)\|f\|_{p, q, \nu}^{p} .
$$

Proof. Note that $\left\|\chi_{Q}\right\|_{p, q, \mu}=\mu(Q)^{1 / p}$. It is easy to verify that

$$
M_{p q} f(x)=\left[M_{r, 1} f^{q}(x)\right]^{1 / q}, \quad r=p / q,
$$

and thus we need only show that $\mu\left\{x: M_{r, 1} f(x)>y\right\} \leqslant\left(C / y^{r}\right)\|f\|_{r, 1, \nu}^{r}$. Note that $\left\|f \chi_{Q}\right\|_{r, 1, \nu} \sim \int f \chi_{Q} \psi_{Q} d \nu$ for some $\psi_{Q}$ with $\left\|\psi_{Q}\right\|_{r^{\prime}, \infty, \nu}=1$ or $\psi_{Q, \nu}^{*}(t) \leqslant 1 / t^{1 / r^{\prime}}$. Hence, if $\phi_{Q}=\chi_{Q} \psi_{Q} / \mu(Q)^{1 / r}$, then $\mu(Q) \phi_{Q, \nu}^{*}(\mu(Q) t) \leqslant 1 / t^{1 / r^{\prime}}$, from which $\Phi \in L\left(r^{\prime}, \infty\right)$.

For the usual Hardy-Littlewood maximal operator, $\Phi \in L\left(p^{\prime}, \infty\right)$ actually characterizes the restricted weak type behavior. If $(u, v)$ is a pair of weights,

$$
\phi_{Q}(x)=\frac{1}{|Q|} \frac{\chi_{Q}(x)}{v(x)}, \quad d \mu=u d x, \quad d \nu=v d x .
$$

then $\sup \int f \phi_{Q} d \nu=\sup |Q|^{-1} \int_{Q} f d x=M f(x)$. Let $\Phi(t)=\sup _{Q}\left\{\mu(Q) \phi_{Q, \nu}^{*}(\mu(Q) t)\right\}$.

Theorem 7. Let $1<p<\infty$. Then $\|M f\|_{p, \infty, \mu} \leqslant C\|f\|_{p, 1, \nu}$ if and only if $\Phi \in$ $L\left(p^{\prime}, \infty\right)$.

Proof. We need only show that the norm inequality implies $\Phi \in L\left(p^{\prime}, \infty\right)$. We use the technique of [3] and choose $f \geqslant 0$ with $\|f\|_{p, 1, v}=1$ and $\int_{Q} f=\int f\left(\chi_{Q} v^{-1}\right) v$ $\geqslant C\left\|\chi_{Q} v^{-1}\right\|_{p^{\prime}, \infty, v}$. For $x \in Q$ we have $M f(x) \geqslant(C /|Q|) \int_{Q} f$. Our assumption is $\mu\{x: M f(x)>y\} \leqslant\left(C / y^{p}\right)\|f\|_{p, 1, v}^{p}$ and, hence, we get, with $y=|Q|^{-1} \int_{Q} f$,

$$
\mu(Q) \leqslant C\|f\|_{p, 1, v}^{p}\left(\frac{1}{|Q|} \int_{Q} f\right)^{-p} \leqslant \frac{2 C|Q|^{p}}{\left\|\chi_{Q} v^{-1}\right\|_{p^{\prime}, \infty, v}^{p}} .
$$

From this it follows that

$$
\frac{\mu(Q)}{|Q|}\left(\frac{\chi_{Q}}{v}\right)_{\nu}^{*}(\mu(Q) t) \leqslant \frac{\mu(Q)^{1 / p}}{|Q|} \frac{1}{t^{1 / p^{\prime}}}\left\{\sup _{\tau>0} \tau^{1 / p^{\prime}}\left(\frac{\chi_{Q}}{v}\right)_{\nu}^{*}(\tau)\right\} \leqslant C / t^{1 / p^{\prime}},
$$

and $\Phi \in L\left(p^{\prime}, \infty\right)$.

6. In this section we will present two generalizations of Theorem 1 to abstract measure spaces.

(i) Consider $(X, \mathfrak{T}, \mu, \nu)$ and a measurable map $T: X \rightarrow \mathbf{R}^{n}$ such that $\mu\left(T^{-1}(Q)\right)$ $<\infty$ for every cube $Q \subset \mathbf{R}^{n}$. Associate with each cube $Q$ a measurable function $\phi_{T^{-1}(Q)}: X \rightarrow[0, \infty)$, with $\operatorname{supp} \phi_{T^{-1}(Q)} \subset T^{-1}(Q)$, and define, for $f: X \rightarrow[0, \infty]$ measurable, the maximal operator

$$
\Re f(x)=\sup \int f \phi_{T^{-1}(Q)} d \nu
$$

where the sup is extended over all cubes $Q$ with center $T(x)$. 
THEOREM 8.

$$
(\vartheta \pi f)_{\mu}^{*}(\xi) \leqslant A \int_{0}^{\infty} \Phi(t) f_{\nu}^{*}(t \xi) d t
$$

where

$$
\Phi(t)=\sup _{Q}\left\{\mu\left(T^{-1}(Q)\right)\left(\phi_{T^{-1}(Q)}\right)_{\nu}^{*}\left(\mu\left(T^{-1}(Q)\right) t\right)\right\} .
$$

For the proof choose for each $x \in E_{\tau}=\{x$ : $\Re f(x)>\tau\}$, a cube $Q_{x}$ centered at $T(x)$ for which $\int f \phi_{T^{-1}\left(Q_{x}\right)} d \nu>\tau$. The Besicovitch covering theorem gives us a countable collection $\left\{Q_{j}\right\}$ with $T\left(E_{\tau}\right) \subset \cup Q_{j}, \Sigma \chi_{Q_{1}} \leqslant C$. Now proceed exactly as in the proof of Theorem 1, replacing $\mu\left(Q_{j}\right)$ there by $\mu\left(T^{-1}\left(Q_{j}\right)\right)$.

(ii) We will again consider an abstract measure space $(X, \mathfrak{N}, \lambda)$ and a measurable map $T: X \rightarrow \mathbf{R}^{n}$ with $\lambda\left(T^{-1}(Q)\right)<\infty, Q$ cube in $\mathbf{R}^{n}$. Let $\nu \geqslant 0$ be a measure on $\mathbf{R}^{n}$ and associate with each cube $Q \subset \mathbf{R}^{n}$ a $\nu$-measurable function $\phi_{Q}$ with supp $\phi_{Q} \subset Q$. For $f: \mathbf{R}^{n} \rightarrow[0, \infty]$ and $x \in X$ we define

$$
M_{T} f(x)=\sup \int f \phi_{Q} d \nu,
$$

where again the sup is extended over all cubes centered at $T(x)$. As in Theorem 8 one can establish

$$
\left(M_{T} f\right)_{\lambda}^{*}(\xi) \leqslant A \int_{0}^{\infty} \Phi(t) f_{\nu}^{*}(t \xi) d t
$$

where

$$
\Phi(t)=\sup _{Q}\left\{\lambda\left(T^{-1}(Q)\right) \phi_{Q, \nu}^{*}\left(\lambda\left(T^{-1}(Q)\right) t\right)\right\} .
$$

As an application we consider $\left(\mathbf{R}^{n}, \mu, \nu\right)$ as in Theorem 1 and assume that $\Phi_{0}(t)=\sup _{Q}\left\{\mu(Q) \phi_{Q, \nu}^{*}(\mu(Q) t)\right\}$ is in $L\left(p^{\prime}, 1\right)$ for some $1<p<\infty$. Then we have $\|M f\|_{p, \mu} \leqslant A\|f\|_{p, \nu}$, where $M f(y)=\sup \int f \phi_{Q} d \nu, Q$ centered at $y$.

TheOREM 9. If $\lambda\left(T^{-1}(Q)\right) \leqslant C \mu(Q), Q \subset \mathbf{R}^{n}$, then $\Phi(t) \in L\left(p^{\prime}, 1\right)$, and hence $\left\|M_{T} f\right\|_{p, \lambda} \leqslant A_{p}\|f\|_{p, \nu}$.

Proof. We simply observe that

$$
\begin{aligned}
\lambda\left(T^{-1}(Q)\right) \phi_{Q, \nu}^{*}\left(\lambda\left(T^{-1}(Q)\right) t\right) & \leqslant \frac{1}{t} \int_{0}^{C \mu(Q) t} \phi_{Q, \nu}^{*}(\tau) d \tau \\
& =\frac{1}{t} \mu(Q) \int_{0}^{C t} \phi_{Q, \nu}^{*}(\mu(Q) \tau) d \tau \leqslant C \frac{1}{C t} \int_{0}^{C t} \Phi_{0}(\tau) d \tau \\
& =C \Phi_{0}^{* *}(C t) \quad
\end{aligned}
$$

REMARK. The above hypothesis is a type of Carleson measure condition [1].

\section{BIBLIOGRAPHY}

1. L. Carleson, Interpolation by bounded analytic functions and the corona problem, Ann. of Math. (2) 116 (1966), 135-157.

2. R. A. Hunt, On L( $p, q)$-spaces, Enseignement Math. 12 (1966), 249-275.

3. H. M. Chung, R. A. Hunt and D. S. Kurtz, The Hardy-Littlewood maximal function on L $(p, q)$-spaces with weights, Indiana Univ. Math. J. 31 (1982), 109-120. 
4. W. B. Jurkat and J. L. Troutman. Maximal inequalities related to a.e. continuity, Trans. Amer. Math. Soc. 252 (1979), 49-64.

5. R. Kerman, Restricted weak type inequalities with weights.

6. M. A. Leckband and C. J. Neugebauer, $A$ general maximal operator and the $A_{p}$-condition. Trans. Amer. Math. Soc. 275 (1983), 821-831.

7. B. Muckenhoupt, Weighted norm inequalities for the Hardy maximal function. Trans. Amer. Math. Soc. 165 (1972), 207-226.

8. B. Muckenhoupt and R. Wheeden. Two weight function norm inequalities for the Hardy-Littlewood maximal function and the Hilbert transform, Studia Math. 55 (1976), 279-294.

9. E. M. Stein, Editor's note: The differentiability of functions in $\mathbf{R}^{n}$, Ann. of Math. (2) 113 (1981), $383-385$.

10. . Singular integrals and differentiability properties of functions, Princeton Univ. Press, Princeton, N.J., 1970.

Department of Mathematics, Purdue University, West Lafayette, Indiana 47907 (Current address of C. J. Neugebauer)

Current address (M. A. Leckband): Department of Mathematical Sciences, Florida International University, Miami, Florida 33199 